\section{Checklist of freshwater fishes collected from Ernakulam District, Kerala, India}

\section{K.S. Jameela Beevi ${ }^{1}$ \& A. Ramachandran ${ }^{2}$}

${ }^{1}$ Department of Zoology, Maharaja's College, Ernakulam, Cochin, Kerala 682011, India

${ }^{2}$ School of Industrial Fisheries, Cochin University of Science and Technology, Cochin, Kerala 682016, India

Email: ${ }^{1}$ ksjameela@yahoo.com; ${ }^{2}$ ram-alappat@eth.net

Aquarium fishes have gained much attention in recent years. Kerala has immense developmental potential in aquarium fishery. Many ichthyological surveys are going on to study the fish fauna of our rivers. The NBSAP (2002) has reported the presence of 159 freshwater fishes in Kerala. Though district wise and river wise diversity studies are going on in Kerala, not much has been recorded on the fresh water fishes in Ernakulam District, Kerala. With this paucity in mind, an attempt has been made to document the freshwater fishes in the rivers of Ernakulam District, Kerala. The systematics of the collected fishes was also studied.

Eleven sampling stations were selected, viz, Angadikkadavu (Station-I) and Chalakkudythode (Station-II) in Chalakkudy River; Ooramana in Muvattupuzha (StationIII), Peruvamuzhy (Station-IV), Vellore near Piravom (Station$\mathrm{V}$ ) and Thengode near Idachira, Kakkanad (Station-VI) in Muvattupuzha River; Aluva (Station-VII), Madathumoola near Desom (Station-VIII), Parambepalam (Station-IX), Kalady (Station-X) and Panieli (Station-XI) in Periyar. The streams and channels opening to the various centres were also included for collecting samples.

Monthly collections were made for a period of two years from January 2000 to December 2001. Fishes were collected using cast and scoop nets

Standard methods (Nelson 1984; Talwar \& Jhingran 1991; Jayaram 1999) were used for the classification and identification

Date of publication (online): 26 September 2009

Date of publication (print): 26 September 2009

ISSN 0974-7907 (online) | 0974-7893 (print)

Editor: M. Arunachalam

\section{Manuscript details:}

Ms \# 01559

Received 19 April 2006

Final received 11 April 2008

Finally accepted 12 September 2009

Citation: Beevi, K.S.J. \& A. Ramachandran (2009). Checklist of freshwater fishes collected from Ernakulam District, Kerala, India. Journal of Threatened Taxa 1(9): 493-494.

Copyright: ๑ K.S. Jameela Beevi \& A. Ramachandran 2009. Creative Commons Attribution 3.0 Unported License. JoTT allows unrestricted use of this article in any medium for non-profit purposes, reproduction and distribution by providing adequate credit to the authors and the source of publication.

Acknowledgements: The authors are grateful to the Head of School of Industrial Fisheries, Cochin University of Science and Technology for giving the necessary facilities for the completion of this work. They extend their sincere gratitude to Dr. K. Rema Devi, Scientist, ZSI, Southern Regional Station, Chennai, for confirming their identification of the species. KSLB extends her sincere gratitude to the UGC for giving her Teacher Fellowship.

\section{OPEN AGCESS | FREE DOWNLOAD

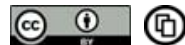

of fishes. The identification was further confirmed with the Zoological Survey of India.

The study indicates that the rivers in

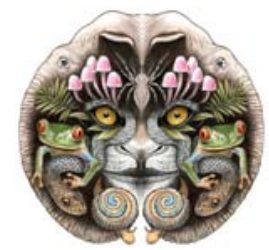
Ernakulam are rich in fish diversity. 69 species were collected during the study. Based on their availability and abundance in the collection, they were classified in to abundant species (*****), common species (***), rare species (**), very rare species (*) and their absence by "O". There were 17 abundant species, 13 common species, 31 rare species and nine very rare species in the study. The systematic positions of the fishes collected during the study are shown in Table: 1. Of the collected fishes, 35 species have high demand in the domestic and international ornamental fish market. These fishes are marked with an asterisk mark in their serial numbers

\section{References}

Nelson, J.S. (1984). Fishes of the World. John Wiley and Sons, New York, $523 \mathrm{pp}$.

Talwar P.K. \& A.G. Jhingran (1991). Inland Fishes of India and Adjacent Countries Vol.1 and 2, Oxford and IBH publishing Co. Pvt. Ltd, New Delhi, $1158 \mathrm{pp}$.

Jayaram, K.C. (1999). The Fresh Water Fishes of the Indian Region. Narendra Publishing House, Delhi, $551 \mathrm{pp}$

NBSAP (2002). Executive Summaries of National Biodiversity Strategy and Action Plans (Ecoregions, States, Sub states, sites, and themes and sub thematic Reviews). December, 2002: 94-04.

Table 1. List of freshwater fishes collected from the rivers of Ernakulam District, Kerala.

Abundance in rivers

CHA MUV PER

Family Cyprinidae

1\# Amblypharyngodon melletinus (Valenciennes) Barilius bakeri Day

Barilius canarensis (Jerdon)

Barilius gatensis (Valenciennes)

Chela dadiburjori (Menon)

Chela fasciata Silas

Danio aequipinnatus (Mc Clelland)

Danio fraseri (Hora and Mukerji)

Danio malabaricus (Jerdon)

Esomus malabaricus Day

Garra gotyla stenorhynchus (Jerdon)

Garra mullya (Sykes)

Gonoproktopterus curmuca(Hamilton-Buchanan)

Gonoproktopterus thomassi (Day)

Horadandia attukorali Deraniyagala

Parluciosoma daniconius (Hamilton-Buchanan)

Puntius amphibius (Valenciennes)

Puntius chola (Hamilton-Buchanan)

Puntius crescentus Yazdani \& Singh

Puntius denisonii (Day)

Puntius fasciatus (Jerdon)

Puntius filamentosus (Valenciennes)

Puntius melanostigma (Day)

Puntius muvattupuzhaensis Jameela

Beevi \& Ramachandran

Puntius parrah Day

Puntius punctatus Day

$7^{\#} \quad$ Puntius sarana sarana (Hamilton-Buchanan)

Puntius sarana subnasutus (Valenciennes)

Puntius vittatus Day

Salmostoma acinaces (Valenciennes)

Salmostoma boopis (Day)

\begin{tabular}{|c|c|c|}
\hline$* * * *$ & $\star * * *$ & $\star \star \star * \star$ \\
\hline$\star *$ & $* *$ & ** \\
\hline * & ** & $\star *$ \\
\hline 0 & $\star *$ & $\star *$ \\
\hline$\star *$ & $* *$ & ** \\
\hline$\star \star *$ & $* *$ & $\star *$ \\
\hline$* * * *$ & $\star \star \star \star$ & $\begin{array}{l}* * * * \\
*\end{array}$ \\
\hline$* * * *$ & $* * * *$ & $\star \star \star * *$ \\
\hline 0 & * & 0 \\
\hline 0 & * & * \\
\hline$\star \star$ & $* *$ & $\star *$ \\
\hline$\star *$ & $* *$ & $\star *$ \\
\hline$\star *$ & 0 & ** \\
\hline$* * * *$ & $* * * *$ & $* * * *$ \\
\hline$* * * *$ & $\star * * *$ & $* \star * *$ \\
\hline$\star * \star$ & $\star \star *$ & $* * *$ \\
\hline$\star *$ & $* *$ & $\star \star$ \\
\hline$* * *$ & $* * *$ & 0 \\
\hline 0 & 0 & $\star *$ \\
\hline$\star \star$ & $* *$ & $\star \star$ \\
\hline$* \star * *$ & $\star * * *$ & $\star \star \star * *$ \\
\hline 0 & $\star \star$ & $\star *$ \\
\hline 0 & $\star *$ & $* *$ \\
\hline$\star \star$ & ** & $\star *$ \\
\hline ** & $\star \star$ & ** \\
\hline$\star *$ & $\star *$ & $* *$ \\
\hline$\star * *$ & $* * *$ & $* * *$ \\
\hline$* * * *$ & $\star \star * *$ & $\star * * *$ \\
\hline ** & $\star \star$ & $\star *$ \\
\hline$\star *$ & $* *$ & $\star \star$ \\
\hline
\end{tabular}

Family Balitoridae

32 ${ }^{\# \quad M e s o n e m a c h e i l u s ~ t r i a n g u l a r i s ~ D a y ~}$ 
Family Cobitidae

33" Lepidocephalus thermalis (Valenciennes)

Family Bagridae

$\begin{array}{ll}34^{\#} & \text { Horabagrus brachysoma (Gunther) } \\ 35 & \text { Mystus armatus (Day) } \\ 36^{\#} & \text { Mystus malabaricus (Jerdon) } \\ 37^{\#} & \text { Mystus montanus (Jerdon) } \\ 38 & \text { Mystus vittatus (Bloch) } \\ & \\ \text { Family Siluridae } \\ 39^{\#} & \text { Ompok bimaculatus (Bloch) } \\ 40 & \text { Ompok malabaricus (Valenciennes) } \\ 41^{\#} & \text { Wallago attu (Bloch \& Schneider) }\end{array}$

Family Heteropneustidae

$42^{\#} \quad$ Heteropneustes fossilis (Bloch)

Family Hemirhampidae

43 Hyporhampus limbatus (Valenciennes)

44 Hyporhampus xanthopterus (Valenciennes)

Family Belonidae

45 Xenetodon cancila (Hamilton-Buchanan)

46 Strongylura strongylura (van Hasselt)

Family Aplocheilidae

47" Aplocheilus blocki (Arnold)

48* Aplocheilus lineatus (Valenciennes)

49" Aplocheilus panchax (Hamilton \& Buchanan)

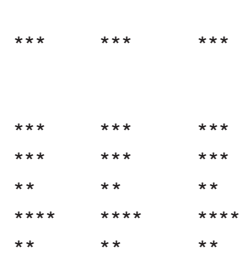

Family Chandidae (Ambassidae)

50 Chanda nama Hamilton \& Buchanan

51" Parambassis dayi (Bleeker)

52" Parambassis thomassi (Day)

Family Nandidae

53 Nandus nandus (Hamilton \& Buchanan)

Family Cichlidae

54" Etroplus maculatus (Bloch)

55" Etroplus suratensis (Bloch)

Family Belontidae

56" Pseudosphromenus cupanus (Valenciennes)

57" Pseudosphromenus dayi Engmann

Family Anabantidae

58" Anabas testudineus (Bloch)

Family Gobiidae

59 Awaous stamineus (Valenciennes)

60 Glossogobius giuris (Hamilton-Buchanan)

$\begin{array}{lll}* * * & * * * & * * * \\ * * * & * * * & * * * \\ * & 0 & 0\end{array}$

* 00

** * *

**

** $* *$

*** $\quad * * * \quad * * *$

00 *

$\star * * * \quad * * * * \quad * * * *$

$\begin{array}{lll}* \star * * & \star * * * & * * * * \\ * * * * & \star * * * & * * * *\end{array}$

** ** * **

* $\quad * * \quad * *$

** $* *$

**** $\quad * * *$

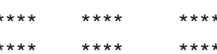

*****

$\begin{array}{lll}* * * * & * * * * & * * * * \\ * * * * & * * * * & * * * *\end{array}$

$\star * * \quad * * * \quad * * *$

** $\quad * * \quad$ *

$\star * * * \quad * * * * \quad * * * *$

Family Channidae

61" Channa gachua (Hamilton \& Buchanan)

62 Channa marulius(Hamilton-Buchanan)

$63^{\# \quad C h a n n a ~ o r i e n t a l i s ~ B l o c h ~ \& ~ S c h n e i d e r ~}$

64 Channa striatus (Bloch)

** * * * * *

** $* * *$

$\begin{array}{lll}* * * & * * * & * * * \\ * * * & * * * & * * *\end{array}$

Family Synbranchidae

65 Ophisternon bengalense Mc Clelland

Family Mastacembelidae

66" Mastacembelus armatus (Lacepede)

67 Mastacembelus guentheri (Day)

Family Clupeidae

68 Dayella malabarica (Day)

* *

** $\quad$ ** * * $\quad * *$

$0 \quad 0$ *

Family Tetraodontidae

69" Tetraodon travancoricus Hora and Nair $\quad * * * * \quad * * * * \quad * * * *$

CHA - Chalakkudy River; MUV - Muvattupuzha River; PER - Periyar River; ${ }^{\star \star \star *}$ - Very common; ${ }^{* \star *}$ - Common; ${ }^{\star *}$ - Rare; ${ }^{*}$ - Very rare; ${ }^{*}$ - in heavy trade

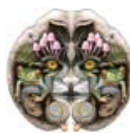

